Methods: This retrospective cohort study used the EGB (Echantillon Généraliste des Bénéficiaires) database which is a $1 \%$ random and representative sample of the French national Health insurance system. EGB contains anonymous demographic and comprehensive medical data on conditions with long-term disease (LTD) status, hospitalizations and reimbursement claims for medications dispensed in the community. The study used the data collected between January 1 , 2007 and December 31, 2015. Inclusion criteria were: 1 ) age $\geq 50$ years; 2 ) hospitalisation for GCA or LTD status for GCA (ICD-10 codes: M31.5/6); and 3) at least 4 drug dispensing of oral GCs within 6 months around the index date. The index date was defined as the date of 1 st occurrence of GCA code and cases were considered as incident if the GCA code first occurred after $\geq 2$ years of follow-up. Demographics, co-morbidities, diagnostic tests and therapies were analysed. A treatment sequence was defined as the start of a new drug or the resumption of the same drug after a stop $\geq 3$ months. Annual incidence was calculated by using the people recorded in the EGB as denominator.

Results: Among the 752717 pts recorded in the EGB, 241 pts fulfilled our criteria. Around 24 pts were newly diagnosed/year with an annual incidence of 7 to 10 / 100,000 people $\geq 50$ years-old. $72 \%$ of the 241 pts were females, mean age was $77.5( \pm 8.9)$ years, mean follow-up $3.7( \pm 2.6)$ years. In the 12 months before index date, $74.3 \%$ of the pts had at least 1 proxy for hypertension, $39.4 \%$ for depression/ insomnia and $33.6 \%$ for osteoporosis. After index date, temporal artery biopsy (TAB) was performed in $43.2 \%$, high-resolution Doppler ultrasound in $35.3 \%$ and positron emission tomography (PET) in $11.6 \%$. Among the 235 pts $(97.5 \%)$ who had at least 1 drug dispensing of oral GCs, 198 pts (84.3\%) used only GCs while $37(16.7 \%)$ also received 1 to 3 adjunctive agents. Mean 1 st GCs sequence duration was 17.2 months $( \pm 16.5)$ in $96.6 \%$. 95 pts $(40.4 \%)$ had a 2 nd sequence, i.e. resume GCs and or start a new drug for a duration of 6.7 months $( \pm 8.1)$ for GCS alone or 12.2 months $( \pm 8.8)$ for GCs+adjunctive drug. The most prescribed GCssparing agent was methotrexate (12.0\%). Others were marginal: hydroxychloroquine 7 pts, azathioprine 4, cyclophosphamide 1, infliximab 1, adalimumab 2 and etanercept 1 pt.

Conclusions: These real-world data indicate an incidence of GCA in France of 7 to 10 cases $/ 100,000$ people $\geq 50$ years-old and underline that most patients with GCA are treated with GCs alone whereas adjunctive agents, mainly methotrexate, are given to $17 \%$ of patients. The utilisation of $T A B$ in only half of the patients might reflect a shift towards increasing use of imaging techniques to diagnosed GCA.

Disclosure of Interest: V. Devauchelle Pensec: None declared, E. Hachulla: None declared, M. Paccalin: None declared, S. Gandon Employee of: Roche SAS, I. Idier Employee of: Chugai Pharma France, M. Nolin: None declared, M. Belhasen: None declared, A. Mahr: None declared

DOI: 10.1136/annrheumdis-2018-eular.3652

\section{FRI0519 WORK PRODUCTIVITY IS IMPAIRED IN PATIENTS WITH BEHÇET'S SYNDROME}

N. Serin, Y. Ozguler, S.N. Esatoglu, V. Hamuryudan, G. Hatemi. Istanbul University Cerrahpasa Medical Faculty, Rheumatology, Istanbul, Turkey

Background: Behçet's syndrome (BS) is most active during young adulthood and working years, thus affecting productivity. Work disability was previously reported especially among BS patients with eye, vascular and joint involvement. Objectives: In this study, we aimed to evaluate the work productivity and instability of patients with BS compared to ankylosing spondylitis (AS) patients and healthy controls $(\mathrm{HC})$

Methods: $100(80 \mathrm{M} / 20 \mathrm{~F})$ consecutive BS patients who were routinely followed in our dedicated BS centre were studied. Patients with $A S ;{ }^{26} 22 \mathrm{M} / 4 \mathrm{~F}$ ) who were followed in the rheumatology outpatient clinic of our unit and $\mathrm{HC}$; ${ }^{30} 18 \mathrm{M} / 12 \mathrm{~F}$ ) were included as controls. Work Productivity and Activity Impairment Questionnaire (WPAI), Work Productivity Survey (WPS), Work Instability Scale (WIS) were used. Quality of life was assessed with the Behçet Disease Quality of Life (BDQoL) scale and disease activity with the Behçet's Disease Current Activity Index.

Results: Table 1 shows the characteristics of the included subjects. 28 of BS patients with only mucocutaneous, 28 with eye, 23 with vascular and 21 with neurologic involvement were included. Among BS patients $41 \%$ reported missing work days (mean 1.4 days $/ \mathrm{mo}$ ), and $49 \%$ reported that their productivity was reduced at least by half (mean 4.1 days/mo). The mean WIS score was 11.7 (5.8) in BS patients. 52 BS patients had moderate and 14 BS patients had high work instability. Patients with BS had significantly higher absenteeism (8.4\% vs. $1.7 \%)$, presenteeism ( $37.6 \%$ vs. 9.3\%), and daily activity impairment ( $27.5 \%$ vs. $8.6 \%)$ than HCs $(p<0.001)$ assessed by WPAI. Scores were similar between BS and AS patients. WIS and WPS scores were also similar between BS and AS patients and worse than healthy controls. Work impairment was more pronounced in patients with eye involvement compared to mucocutaneous involvement $(p=0.006)$ and there were no differences between other BS groups. The WPAI presenteeism score was moderately correlated with Behçet Disease Quality of Life scale score $(r=-0.57)$. Multivariate analysis showed that $\mathrm{QoL}(\mathrm{OR}=0.77,95 \% \mathrm{Cl}=0.66-0.88)$ and disease activity $(\mathrm{OR}=1.66,95 \% \mathrm{Cl}=1.01-2.50)$ were related with WPAIpresenteeism.

Abstract FRI0519 - Table 1. Characteristics of the included subjects

\begin{tabular}{lccc}
\hline & $\begin{array}{c}\text { Behçet's } \\
\text { syndrome } \\
(\mathrm{n}=100)\end{array}$ & $\begin{array}{c}\text { Ankylosing } \\
\text { spondylitis } \\
(\mathrm{n}=26)\end{array}$ & $\begin{array}{c}\text { Healthy } \\
\text { controls } \\
(\mathrm{n}=30)\end{array}$ \\
\hline Male, $\mathrm{n}(\%)$ & $80(80)$ & $22(85)$ & $18(60)$ \\
Mean (SD) current age, & $36 \pm 7.8$ & $36 \pm 6.4$ & $31 \pm 8.1$ \\
Mean (SD) disease duration & $8 \pm 5.5$ & $7.46 \pm 4.6$ & $\mathrm{~N} / \mathrm{A}$ \\
WIS (mean \pm SD) & $12 \pm 6$ & $11 \pm 5.5$ & $6 \pm 4$ \\
WPAl-Absenteeism & $8 \pm 15$ & $8 \pm 19$ & $2 \pm 7$ \\
(mean \pm SD) & & & \\
WPAI-Presenteeism (mean & $38 \pm 29$ & $32 \pm 26$ & $9 \pm 21$ \\
$\pm S D)$ & & & \\
WPS-Absenteeism & $1.4 \pm 3$ & $1.2 \pm 3$ & $0.1 \pm 0.3$ \\
(mean $\pm S D)$ & & & \\
WPS -Presenteeism (mean & $4 \pm 7$ & $4 \pm 6$ & $1 \pm 3$ \\
$\pm S D)$ & & & \\
BDCAF (mean $\pm S D)$ & $4.47 \pm 2.79$ & $\mathrm{~N} / \mathrm{A}$ & $\mathrm{N} / \mathrm{A}$ \\
BSAS (mean $\pm S D)$ & $21.47 \pm 17.5$ & $\mathrm{~N} / \mathrm{A}$ & $\mathrm{N} / \mathrm{A}$ \\
BDQoL (mean $\pm S D)$ & $19.53 \pm 8.3$ & $\mathrm{~N} / \mathrm{A}$ & $\mathrm{N} / \mathrm{A}$ \\
BASDAl (mean $\pm S D)$ & $\mathrm{N} / \mathrm{A}$ & $3.3 \pm 1.8$ & $\mathrm{~N} / \mathrm{A}$ \\
BASFI (mean $\pm S D)$ & $\mathrm{N} / \mathrm{A}$ & $2.8 \pm 2.9$ & $\mathrm{~N} / \mathrm{A}$ \\
\hline
\end{tabular}

N/A: Not applicable; Behçet Disease Quality of Life: BDQoL; Behçet's Disease Current Activity Index (BDCAI); Behçet's Syndrome Activity Score (BSAS)

Conclusions: Work productivity is impaired in BS patients, especially among those with eye involvement. Work instability is frequent and correlated with disease activity and quality of life.

Disclosure of Interest: None declared

DOI: 10.1136/annrheumdis-2018-eular.6795

FRIDAY, 15 JUNE 2018: Osteoarthritis

\section{FRI0520 DISCOVERY OF POTENTIAL BIOMARKERS FOR THE DIAGNOSIS OF EROSIVE AND NODAL HAND OSTEOARTHRITIS}

M. Camacho Encina ${ }^{1}$, V. Calamia ${ }^{1}$, F. Picchi ${ }^{1}$, I. Ortega ${ }^{2}$, P. Fernández Puente ${ }^{1}$, L. González Rodríguez ${ }^{1}$, C. Ruiz Romero ${ }^{1}$, F.J. Blanco ${ }^{1} .{ }^{1}$ Rheumatology Research Group (GIR), ProteoRed, PRB2-ISCIII, INIBIC-Complejo Hospitalario Universitario de A Coruña (CHUAC), A Coruña; ${ }^{2}$ Proteomics core Facility, PRB2-ISCIII, IMIBICHospital Universitario Reina Sofía, Córdoba, Spain

Background: Two different phenotypes of hand osteoarthritis (HOA) have been defined: nodal hand osteoarthritis (NHOA) and erosive hand osteoarthritis (EHOA). NHOA involve bone enlargement of the underlying interphalangeal joints, which may typically give rise to Heberden's nodes, synovitis and swelling. EHOA is a particularly aggressive form characterised by an abrupt onset, as well as signs of inflammation and subchondral erosions. In the absence of efficient diagnostic methods, searching for specific biomarkers for each subtype may help to characterise them.

Objectives: To define a panel of specific protein markers for the characterisation of EHOA and NHOA and its potential use in clinic.

Methods: A proteomic approach based on peptide labelling with Isobaric tags for relative and absolute quantitation (iTRAQ) was performed using two different sets of sera $(n=55)$. Samples were classified in 4 groups of study (EHOA, $n=10$; nonEHOA, $n=10$; NHOA $n=10$; non-NHOA, $n=5$ ) and 2 control groups (rheumatoid arthritis (RA), $n=10$ and psoriatic arthritis (PSA), $n=10$ ). Serum proteins were digested and peptides from each condition to be compared were differentially labelled with ITRAQ reagents (Sciex). Then, samples were combined and analysed by two-dimensional liquid chromatography coupled to mass spectrometry in a TripleTOF 5600 Mass Spectrometer System (Sciex). Protein identification and quantitation was carried out using ProteinPilot software v.5.0.1.

Results: A total of 257 different proteins were identify with more than two peptides and a total score $\geq 2$ at $95 \%$ confidence. In order to identify specific biomarkers for the characterisation of $\mathrm{NHOA}$ and EHOA phenotypes, each group was compared with the non-NHOA or non-EHOA respectively, and also with the control groups. After all the comparisons were made, 26 unique different proteins were found specific of the nodular phenotype. Vasorin (VAS) showed elevated levels in patients diagnosed with NHOA when compared to non-NHOA, RA and PSA groups. On the other hand, 36 unique proteins were identified in those patients with EHOA. Extracellular matrix protein 1 (ECM1) was found with higher concentrations in EHOA than in non-EHOA, RA and PSA patients. In addition, both HOA phenotypes were compared to the control groups and a panel of 30 different proteins 
were defined. Among these proteins, vascular cell adhesion molecule-1 (VCAM1) was found increased in HOA compared to RA and PSA groups.

Conclusions: A specific protein profile for the characterisation of EHOA and NHOA disorders has been established. VAS showed elevated levels in patients with $\mathrm{NHOA}$, whereas ECM1 was increased in patients diagnosed with the erosive form of the disease. As none of them were identify in the other phenotype, they might be phenotype-specific biomarkers. In addition, VCAM1 was found with higher levels in both phenotypes of HOA when compared with RA and PSA and might be used to differentiate hand osteoarthritis from other rheumatic diseases. Acknowledgements: Financial support (IN606A-2016/012) from the Xunta de Galicia and the European Union (European Social Fund - ESF), is gratefully acknowledged.

Disclosure of Interest: None declared

DOI: 10.1136/annrheumdis-2018-eular.4495

\section{FRI0521 COMBINING FRACTAL- AND ENTROPY-BASED BONE TEXTURE ANALYSIS FOR THE PREDICTION OF OSTEOARTHRITIS: DATA FROM THE MULTICENTER OSTEOARTHRITIS STUDY (MOST)}

R. Ljuhar ${ }^{1}$, Z. Bertalan ${ }^{1}$, S. Nehrer ${ }^{2}$, B. Norman ${ }^{3}$, H.-P. Dimai ${ }^{4}$, A. FahrleitnerPammer $^{4}$, D. Ljuhar ${ }^{3} .{ }^{1}$ Research and Development, ImageBiopsy Lab, Vienna; ${ }^{2}$ Center for Regenerative Medicine and Orthopedics, Danube University, Krems, Krems; ${ }^{3}$ Research and Development, Braincon Technologies, Vienna; ${ }^{4}$ Department of Internal Medicine, Division of Endocrinology and Diabetology, Medical University of Graz, Graz, Austria

Background: Osteoarthritis $(O A)$ is one of the leading causes of long-term pain and disabilities associated with musculoskeletal disorders. Effective treatment and disease-progression slowdown depend on early detection and quantification of risk. However, current disease parameters, like joint space width (JSW), have proven to be insufficient for the prediction of $O A$.

Objectives: The purpose of the present study was to investigate if combining fractal- and entropy-based bone texture analyses with joint space width (JSW) and joint space area (JSA) may improve prediction of OA. Conventional posterioranterior (PA) knee radiographs of men and women were obtained from the Multicenter Osteoarthritis Study (MOST) database, which provides valuable information to identify and define modifiable biomechanical, bone and structural, nutritional, and other risk factors for new disease and progression of existing disease.

Methods: Oriented fractal- and entropy based texture algorithms were developed, using state-of-the-art computer hardware and software as well as specific machine-learning algorithms. The selected subchondral area used for textural analyses included 4 regions of interest $(\mathrm{ROI})$ in the proximal tibia and one on each condyle of the distal femur (figure 1). Furthermore, JSW and JSA were assessed using newly developed and fully automated software.

Results: 1092 conventional knee radiographs obtained from one study centre were screened for eligibility. Of these, a total of 574 radiographs ( 230 women, 344 men) met the inclusion criteria, i.e. a Kellgren \& Lawrence (KL) score of 0 at baseline. At month 84, 41 female and 79 male patients had developed $K L>1$, and 189 female and 265 male patients remained at KLO. Area-Under-the-Curve (AUC) for incident OA using JSW/JSA and clinical features was $0.67 \pm 0.08$ for women, and $0.61 \pm 0.1$ for men. In contrast, combining fractal/entropy-based texture, JSW/A and clinical features resulted in significantly improved AUC for women and men $(0.80 \pm 0.07$ for women and $0.69 \pm 0.1$ for men, respectively). To test whether these differences in predicting incident-OA were significant, we performed classifier comparison: $t=3.84 ; p<10-3$ for women, and $t=3.38 ; p<10-3$ for men.

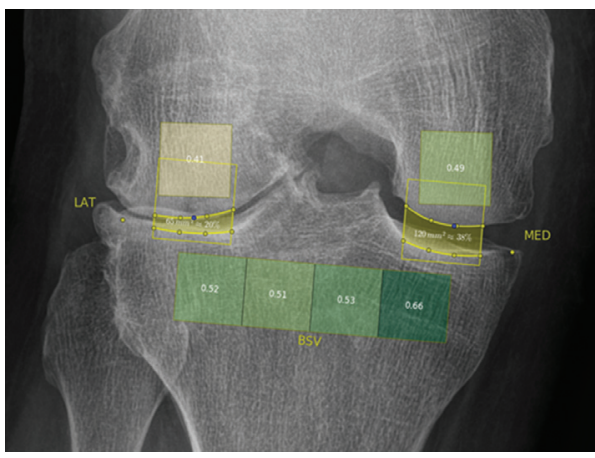

Conclusions: This study provides strong evidence, that a combination of fractaland entropy-based textural analyses of plain subchondral bone radiographs together with JSW/A and clinical features is superior to JSW/A and clinical features alone in predicting incident $\mathrm{OA}$ in men and women.
REFERENCE:

[1] http://most.ucsf.edu/studyoverview.asp

Disclosure of Interest: R. Ljuhar Shareholder of: ImageBiopsy Lab, Z. Bertalan : None declared, S. Nehrer: None declared, B. Norman : None declared, H.-P Dimai : None declared, A. Fahrleitner-Pammer: None declared, D. Ljuhar: None declared

DOI: 10.1136/annrheumdis-2018-eular.1118

\section{FRI0522 VITAMIN D SUPPLEMENTATION IMPROVES DEPRESSION IN KNEE OSTEOARTHRITIS PATIENTS OVER 24 MONTHS}

S. Zheng ${ }^{1}$, L. Tu${ }^{2}$, F. Cicuttini ${ }^{3}$, W. $\operatorname{Han}^{1}$, Z. Zhu ${ }^{1}$, B. Antony ${ }^{1}$, A. Wluka ${ }^{3}$, T. Winzenberg ${ }^{1,4}$, D. Aitken ${ }^{1}$, L. Blizzard ${ }^{1}$, G. Jones ${ }^{1}$, C. Ding ${ }^{1,5}$. ${ }^{1}$ Menzies Institute for Medical Research, University of Tasmania, Hobart, Australia; ${ }^{2}$ Department of Rheumatology, The third Affiliated Hospital of SUN YAT-SEN University, Guangzhou, China; ${ }^{3}$ Department of Epidemiology and Preventive Medicine, Monash University, Melbourne; ${ }^{4}$ Faculty of Health, University of Tasmania, Hobart, ${ }^{5}$ Clinical Research Centre, Zhujiang Hospital, Southern Medical University, Guangzhou, Australia

Background: Although depression is prevalent in osteoarthritis (OA) patients and the positive association between vitamin $\mathrm{D}$ deficiency and depression has been demonstrated, no study has examined the effect of vitamin D supplementation on depression in OA patients.

Objectives: To determine the effect of vitamin D supplementation and maintain ing sufficient serum vitamin $D$ on depression in patients with knee $O A$ and vitamin $D$ deficiency.

Methods: Participants with symptomatic knee OA and vitamin D deficiency were enrolled in a randomised, placebo-controlled trial and received 50,000IU vitamin $D_{3}(n=209)$ or placebo $(n=204)$ monthly for 24 months. Serum 25 -hydroxyvitamin D $[25(\mathrm{OH}) \mathrm{D}]$ was measured at baseline, month 3 and 24. Depression was measured using the patient health questionnaire (PHQ-9), and knee symptoms were assessed using Western Ontario and McMaster Universities Arthritis Index (WOMAC) at baseline, month 3, 6, 12 and 24. Multilevel mixed-effect models were used to estimate the association between exposures and outcomes adjusting for potential confounders.

Results: Over 24 months, 340 participants (82.3\% retention rate) completed the study. The prevalence and incidence of depression were $25.4 \%$ and $11.2 \%$, respectively. Depression improved more in the vitamin $\mathrm{D}$ supplementation group ( $\beta$ : $-0.45,95 \% \mathrm{Cl}:-0.84$ to -0.07 ) compared to the placebo group ( $\beta$ : 0.21 $95 \% \mathrm{Cl}:-0.19$ to 0.61$)(\mathrm{p}=0.02)$ and in those participants who maintained vitamin D sufficiency ( $\beta$ : $-0.44,95 \% \mathrm{Cl}:-0.88$ to -0.00 ) compared to those who did not maintain sufficiency $(\beta: 0.40,95 \% \mathrm{Cl}$ : -0.18 to 0.97$)(p=0.02)$ over 24 months.

Abstract FRI0522 - Table 1. Effects of vitamin D supplementation over 24 months on change in PHQ-9

\begin{tabular}{lccc}
\hline & $\begin{array}{c}\text { Mean change, } \\
(95 \% \mathrm{Cl})\end{array}$ & $\begin{array}{c}\text { Between-group difference change, mean } \\
(95 \% \mathrm{Cl})\end{array}$ & $\begin{array}{c}\mathrm{P} \\
\text { value }\end{array}$ \\
\hline $\begin{array}{l}\text { Placebo } \\
\text { Group }\end{array}$ & $0.21(-0.19$ to 0.61$)$ & $-\mathbf{0 . 6 6}(-\mathbf{1 . 2 2}$ to $-\mathbf{0 . 1 1})$ & $\mathbf{0 . 0 2}$ \\
$(\mathrm{n}=204)$ & & \\
$\begin{array}{l}\text { Vitamin } \mathrm{D} \\
\text { Group }\end{array}$ & $-\mathbf{0 . 4 5 ( - 0 . 8 4}$ to & & \\
$(\mathrm{n}=209)$ & $-\mathbf{0 . 0 7})$ & & \\
\hline
\end{tabular}

Changes in outcomes are generated from mixed-effect models adjusted for age, sex and body mass index.

Abstract FRI0522 - Table 2. Effects of vitamin D status over 24 months on change in PHQ-9

\begin{tabular}{lccc}
\hline & $\begin{array}{c}\text { Mean change, } \\
(95 \% \mathrm{Cl})\end{array}$ & $\begin{array}{c}\text { Between-group difference } \\
\text { change, mean }(95 \% \mathrm{Cl})\end{array}$ & $\begin{array}{c}\mathrm{P} \text { - } \\
\text { value }\end{array}$ \\
\hline Not maintaining sufficient & $0.40(-0.18$ to & $-0.83(-1.56$ to $-\mathbf{0 . 1 1})$ & $\mathbf{0 . 0 2}$ \\
vitamin $\mathrm{D}(\mathrm{n}=114)$ & $0.97)$ & & \\
Maintaining sufficient vitamin & $-\mathbf{0 . 4 4}(-0.88$ to & & \\
$D(n=226)$ & $-\mathbf{0 . 0 0})$ & & \\
\hline
\end{tabular}

Changes in outcomes are generated from mixed-effect models adjusted for age, sex and body mass index. 